\title{
Ovarian Vein Embolization with N-butyl-2 Cyanoacrylate Glubran-2® for the Treatment of Chronic Pelvic Pain Secondary to Pelvic Congestion Syndrome
}

\author{
Nanjing First Hospital \\ Xu He \\ Nanjing First Hospital

\section{Boxiang Zhao} \\ Nanjing First Hospital \\ Jie Kong \\ Nanjing First Hospital \\ Haobo Su \\ Nanjing First Hospital
}

Maofeng Gong ( $\sim$ 305362905@qq.com )

\section{Research}

Keywords: Pelvic congestion syndrome, chronic pelvic pain, ovarian vein, embolization, Glubran-2

Posted Date: June 12th, 2020

DOI: https://doi.org/10.21203/rs.3.rs-34421/v1

License: (c) (i) This work is licensed under a Creative Commons Attribution 4.0 International License. Read Full License 


\section{Abstract}

Objective: To evaluate the safety and efficacy of ovarian vein embolization using N- butyl-2 cyanoacrylate (NBCA) Glubran-

$2{ }^{\circledR}$ in the treatment of chronic pelvic pain (CPP) secondary to pelvic congestion syndrome (PCS).

Materials and Methods: Between January 2013 and June 2019, seven wo-men (mean age, 45.6 615.9 y) presenting with CPP secondary to PCS were evaluated for ovarian vein embolization with Glubran- ${ }^{\circledR}$. PCS was initially diagnosed by transva-ginal Doppler Ultrasound or CT and further confirmed by pelvic venography. Medical database was retrospectively reviewed.

Results: Glubran-2 was employed as a sole embolic material in two cases, four cases subsequently underwent added microcoils in the opening of varicose ovarian vein trunk during the same procedure. Secondary embolization was achieved in one case due to CPP recurrence one month after initial embolization with microcoils. Technical success of ovarian vein embolization with Glubran-2 was achieved in all patients. There were no Glubran-2 related complications mid procedure and postintervention. With mean $62 \pm 40$ months (range, 12-102 months) follow-up, neither persistent nor recurrent CPP was observed. Clinical efficacy was evaluated on the 1st, 3rd, 6th, 12th months, complete or slight improvement of CPP after embolization were achieved in all patients. VAS score used to identify preintervention and postintervention significantly decreased $(p<0.001)$. Two patients after Glubran-2 embolization gave birth to a healthy baby during follow-up.

Conclusions: Ovarian vein embolization with Glubran-2 is a feasible and safe treatment of CPP secondary to PCS. In particularly, it appears to be a potential and attractive alternative when patients with the desires of symptom relief and reproduction.

\section{Key Message}

I There is still no consensus on which embolic materials on preferences to be proposed under the condition of chronic pelvic pain (CPP) secondary to pelvic congestion syndrome (PCS). I Glubran-2 was employed as a sole embolic material or subsequently underwent added microcoils at the opening of varicose ovarian vein trunk during the same procedure. I Ovarian vein embolization with Glubran-2 is a feasible and safe treatment of CPP secondary to PCS. I In particularly, ovarian vein embolization with Glubran-2 appears to be a potential and attractive alternative when patients with the desires of symptom relief and reproduction.

\section{Introduction}

Chronic pelvic pain (CPP) secondary to pelvic congestion syndrome (PCS) is common only presented with a condition of non-cyclic pelvic pain greater than 6 months in duration, typically affecting 39\% women in reproductive age (20-45 years) [1]. This chronic positional pain is variable in term of intensity and duration, is frequently accompanied by dyspareunia and urgent bladder irritability, which can extend to the posteromedial thigh or buttock and has an extremely negative impact on quality of life [2,3]. Despite an increasing awareness of this condition, the precise etiology of CPP secondary to PCS remains uncertain, which is probably related to multifactor. Pelvic vein incompetence with retrograde flow in varicose uteroovarian plexus, has been implicated as one of the most important causes in CPP secondary to PCS [4].

Ovarian vein embolization has been recommended with a 2B level of evidence for the treatment of PCS, with a low rate of morbidity or complications and has largely replaced open surgical intervention [5, 6]. The goal of ovarian vein embolization is to occlude incompetent varicose veins to reduce excessive blood flow within the pelvic vein plexus. As previous reported $[7,8]$, conventional embolic materials used for ovarian vein embolization including sclerosing agents and metal coils, with a clinical efficacy ranging from $58-93 \%$. However, sclerotherapy is associated with a difficult monitoring with fluoroscopy and coils convey a risk of migration, hypersensitivity or further recanalization [7-9]. Whereas, there are still no consensus on which embolic materials on preferences to be proposed under this condition. 
Liquid embolic materials such as N-butyl-2 cyanoacrylate (NBCA) have gained an efficacy for achieving hemostasis in the treatment of acute arterial hemorrhage in various organs [10]. In particular, it appears to have the considerable advantage of high-rate polymerization, making it to be a potential alternative in PCS. Nevertheless, to our knowledge, embolization of PCS with Glubran-2 for the treatment of CPP is rarely described in previously literatures, and consensus on the efficacy and safety of Glubran-2 in this condition have not been reached. The purpose of present study is to evaluate the out-come of embolization with Glubran-2® for controlling CPP secondary to the PCS.

\section{Materials And Methods Study Population}

This retrospective study was approved by both our institutional review board and by the written consent of patients. A search of medical database between January 2013 and June 2019 was performed, two patients who encountered CPP secondary to PCS underwent ovarian vein embolization with Glubran-2 as the primary embolic material were included. In addition, five patients received the embolic material of both Glubran-2 and microcoils during the same procedure were also included. The mean women patient age was $45.6 \pm 15.9$ years and CPP was $26.8 \pm 12.1$ months in duration. Glubran-2 was employed for seven patients, six underwent left unilateral ovarian vein embolization, and one underwent right unilateral ovarian vein embolization. The characteristics are summarized in Table 1. Of included cases, the received ovarian vein embolization with Glubran-2 due to symptoms including CPP (7/7; 100\%), dyspareunia (4/7; 57.1\%), dysmenorrhea (5/7; $71.4 \%)$, vulvar varices $(1 / 7 ; 14.3 \%)$. All patients underwent transvaginal Doppler Ultra-sound to exclude endometriosis or adenomyosis, three cases were confirmed PCS via added enhanced-CT (Fig. 1.) before pelvic venography. 
Table 1

Patients with CPP underwent ovarian vein embolization using the N-butyl-2 cyanoacrylate (NBCA) Glubran-2

\begin{tabular}{|c|c|c|c|c|c|c|c|c|c|}
\hline \multirow{2}{*}{$\begin{array}{l}\text { Patient } \\
\text { No. }\end{array}$} & \multirow[t]{2}{*}{ Age(yr) } & \multirow{2}{*}{$\begin{array}{l}\text { Symptom } \\
\text { duration } \\
\text { (mo) }\end{array}$} & \multirow[t]{2}{*}{ Parity } & \multirow{2}{*}{$\begin{array}{l}\text { Embolization } \\
\text { vein }\end{array}$} & \multirow{2}{*}{$\begin{array}{l}\text { Timing of } \\
\text { NBCA } \\
\text { Glubrans- } \\
2 \text { use }\end{array}$} & \multicolumn{2}{|c|}{ NBCA Glubrans-2 } & \multicolumn{2}{|c|}{ Microcoils $^{a}$} \\
\hline & & & & & & $\begin{array}{l}\text { Concentration } \\
\text { ratio }\end{array}$ & $\begin{array}{l}\text { Volume } \\
(\mathrm{mL})\end{array}$ & Numbers & $\begin{array}{l}\text { Size } \\
(\mathrm{mm})\end{array}$ \\
\hline 1 & 61 & 33 & G5P3 & LOV & First & $1: 3$ & 5 & - & - \\
\hline 2 & 59 & 25 & G4P3 & LOV & First & $1: 4$ & 4 & 4 & $\begin{array}{l}10 \times \\
12\end{array}$ \\
\hline 3 & 31 & 18 & G3P1 & LOV & First & $1: 3$ & 5 & 5 & $\begin{array}{l}10 \times \\
12 \& \\
14 \times \\
10\end{array}$ \\
\hline 4 & 40 & 31 & G3P2 & LOV & First & $1: 3$ & 5 & - & - \\
\hline 5 & 38 & 23 & G2P2 & ROV & Second & $1: 4$ & 3 & 5 & $\begin{array}{l}12 \times \\
14 \& \\
14 \times \\
14\end{array}$ \\
\hline 6 & 65 & 48 & G4P2 & LOV & First & $1: 3$ & 4 & 4 & $\begin{array}{l}14 x \\
6 \& \\
14 x \\
10\end{array}$ \\
\hline 7 & 25 & 10 & G1P1 & LOV & First & $1: 4$ & 3 & 2 & $\begin{array}{l}14 \times \\
10 \& \\
14 \times \\
14\end{array}$ \\
\hline
\end{tabular}

Note: $\mathrm{CPP}=$ chronic pelvic pain, $\mathrm{G}=$ gravidity, $\mathrm{P}=$ parity, $\mathrm{LOV}=$ left ovarian vein, $\mathrm{ROV}=$ right ovarian vein, $\&=$ and

No. 3 and 7 patients gave birth to a healthy baby after embolization

a Underwent microcoils (COOK medical) sealing the opening trunk of ovarian vein

N-butyl-2 Cyanoacrylate Embolization Procedure

The embolic material used in present study was Glubran-2® (N-butyl-2 cyanoacrylate; Braun, Melungeon, Germany), a modified of NBCA Glubran. The benefits and potential risks of embolization with Glubran-2 was explained toward patients and/or their relatives, a detailed informed consent was obtained in all cases.

Under local anesthesia, a 4-French (F) sheath (Radifocus Introducer II Introducer Sheath; Terumo, Leuven, Belgium) was initially inserted into the femoral vein. In order to confirm the diagnosis of varicose ovarian vein and uterine veins engorgement, an overview venography was initial performed before embolization using a 4-F Cobra catheter (Radifocus Angiographic Catheter; Terumo, Leuven, Belgium) along with and without Valsalva maneuver. When incompetent segments of ovarian and uterine veins were identified, a compatible 2.4 F microcatheter (Progreat; Terumo, Leuven, Belgium) was subsequently coaxially positioned, insuring the micro-catheter tip as close as possible into the target varicose veins of the utero-ovarian plexus. The dead space of the microcatheter was initially loaded with $5 \%$ dextrose solution period to prevent early polymerization of Glubran-2 into the lumen of the microcatheter. To improve visualization, Glubran-2 was thoroughly mixed with ethiodized oil (Ethiodized Poppyseed Oil injection; Hengrui Medicine, Jiangsu, China) (concentration ratio ranged from 1:3 to 1:4, depending personally on consideration of distance from the microcatheter tip to the vein site and vein flow speed). Under fluoroscopy control, the mixture was injected as slowly as possible using thumb pressure and was adjusted according to Glubran-2 propagation in the varicose veins and target vein flow speed. Glubran-2 injection was continued until the varicose veins was completely occluded, ensure that avoiding undesired embolization of normal vein branches as 
possible, at the end of Glubran-2 embolization, the microcatheter was removed rapidly, and microcoils (MWCE; COOK, Bjaeverskov, Denmark) were finally employed in the opening of varicose ovarian vein trunk to reduce the risk of non-target embolization from Glubran-2 overflow and a final venography through the Cobra catheter was performed to show veins occlusion.

\section{Definitions of efficacy, safety and Follow-up}

The efficacy of ovarian vein embolization with Glubran-2 included both technical and clinical evaluations [11]. Technical success was defined as a complete occlusion of tar-get incompetent varicose ovarian vein and reflux pelvic veins on final venography. CPP in preintervention, postintervention and follow-up was quantified from 0 to 10 accord-ing to Visual Analog Scale (VAS) score and was divided into four categories: no pain (0-1), mild pain (2-4), moderate pain (5-7), and severe pain (8-10) [12]. Clinical symptom improvement was divided into three categories: complete, when VAS score decreased to 0-1; slight, when the level of pain reduced one or two categories; no improvement, when VAS score stayed in the same category or worsened. Clinical success was defined as complete or slight improvement of the clinical symptom of CPP, without needed to repeat endovascular treatment or surgery. The safety was evaluated including complications both mid Glubran-2 embolization and postintervention, especially adverse events mid Glubran-2 injection. During follow-up, clinical efficacy evaluation according to the VAS score was performed by telephone for all patients on the 1st, 3rd, 6th months and at 6-month intervals thereafter. Any instances of increased category of CPP, a consultation was proposed for a clinical examination (transvaginal Doppler Ultrasound or enhanced-CT).

\section{Statistical Analyses}

The SPSS statistical software package (version 23.0; SPSS statistical software, Chicago, Illinois, USA) was used for all statistical analyses in this study. Continuous variables were expressed as the (means \pm standard deviation). Qualitative variables were presented as a percentage. When assessing the correlation between preintervention and post intervention variables, a paired $t$ test was used and a box plot was achieved. Findings with a $P$ value less than 0.05 were deemed statistically significant.

\section{Results}

Glubran-2 was employed as a sole embolic material in two cases, and four cases with Glubran-2 embolization subsequently underwent added microcoils at the opening of varicose ovarian vein trunk during the same procedure (Fig. 2.), the mean microcoils numbers used were $4 \pm 1$. Secondary embolization was achieved in one case due to CPP recurrence one month after initial embolization with microcoils (Fig. 3.). The distribution of embolization is shown in Table 1. The concentration ratio of Glubran-2 was $1: 3$ to $1: 4$ and the mean volume injected was $4.14 \pm 0.90 \mathrm{~mL}$ (ranged, $3-5 \mathrm{~mL}$ ). Final venography revealed complete occlusion of all targeted varicose veins and technical success of embolization with Glubran-2 was achieved in all patients. There were no Glubran-2 related complications mid procedure and postintervention.

In preintervention, one case experienced mild pain, two cases with moderate pain, and four cases with severe pain. With mean $62 \pm 40$ months (range, 12-102 months) follow-up, neither persistent nor recurrent CPP, which required repeat endovascular treatment or surgery after embolization with Glubran-2, was observed. Clinical efficacy was evaluated on the 1st month [(7.57 \pm 1.81 vs $2.29 \pm 0.76), 95 \% \mathrm{Cl}, 4.257-6.314]$, 3rd months [(7.57 \pm 1.81 vs $1.29 \pm 0.76), 95 \% \mathrm{Cl}, 5.257-$ 7.315], 6th months [(7.57 \pm 1.81 vs $0.86 \pm 0.69), 95 \% \mathrm{Cl}, 5.330-8.100], 12$ th months [(7.57 \pm 1.81 vs $0.42 \pm 0.53), 95 \% \mathrm{Cl}$, 5.789-8.500], complete or slight improvement of CPP after embolization were achieved in all patients. VAS score used to identify preintervention and postintervention significantly decreased $(p<0.001$, showed in Fig. 4.). Of noted, interesting, two young patients after Glubran-2 embolization of the ovarian vein gave birth to a healthy baby during follow-up.

\section{Discussion}


PCS is an underappreciated cause of CPP and disability in young women [3,13]. Living with CPP secondary to PCS is difficult and not only affects the woman directly, but also her interactions with her family and friends, as well her general outlook on life, several treatment modalities of CPP have been proposed with time $[3,6,13,14]$. Conservative treatment can be achieved with psychotropic or non-steroidal anti-inflammatory drugs; however, it provides merely short-term relief of CPP while patients waiting for further investigations or a more permanent treatment but has little role in sustained long-term management [15]. As a surgical alternative, hysterectomy has been eliminated as the first option due to the failure of symptomatic CPP reduction [16]. Open or laparoscopic surgery to ligate the insufficient veins, has historically been proposed as a replaced treatment [17], however, this modality is also rarely performed at present since it has invasiveness, high recurrence and long recovery period. During the current century, endovascular embolization treatment, which involves advantage of minimal invasive-ness and high success rate, has been widely accepted as one of the most effective treatment options $[5-8,11-14,16]$.

During the procedure of ovarian vein embolization, various embolic materials are used, the most commonly involves liquid sclerosing agents and metal coils and various retrospective case series were published on this subject $[7,8,12,18]$. Kim et al. described a cases series with foam sclerosant embolization, the VAS significantly decreased from $7.6 \pm 1.8$ to $2.9 \pm 2.8$ at follow-up of $45 \pm 18$ month, and Laborda et al. reported a group with metal coils embolization, a significant reduction of VAS from $7.34 \pm 0.7$ to $0.78 \pm 1.2$ was similarly observed at follow-up of 5 -year $[12,18]$. Unfortunately, by far, solid data supporting the superiority of one material over another remains lacking. In present study, Glubran-2 achieved a satisfying outcome with VAS score decreased from $7.57 \pm 1.81$ to $0.86 \pm 0.69$ at follow-up of 6 th months, which seems to have a similar efficacy but in a shorter relief time. These may be interpreted to the physicochemical property of Glubran-2, which works independently of hemostatic capacity, polymerization can occur immediately upon contact with blood, leading to instant and complete occlusion of insufficient venous axes. In addition, in order to avoid the risk of embolic material migration, concomitant with/without microcoils following embolization with Glubran-2 at the opening of ovarian veins trunk were performed and no migration event occurred in present study. It should be noted that one case in present study failed to meaningful symptom relief and encountered recurrent CPP after initial microcoils embolization, reasons for this response may be related to an incomplete occlusion of multiple ovarian vein trunks, which account for $24-40 \%$ patients [14]. Finally, embolization with Glub-ran-2 as a second treatment achieved an available CPP relief. Although lacking robust supporting evidence, Glubran-2 appears a potential to treat the multiple small tributaries that are often associated with the ovarian veins and may function as a recurrence source.

The safety is an important component for evaluation of ovarian vein embolization, and complications in such endovascular treatment are reported practically rare $[1,5,6]$. A major complication of ovarian vein embolization is the non-target veins embolization, which may be caused by incorrected concentration ratio glue/coils used or protrusion. Another common complication is the migration of coils or glue fragments, which may be attributed to incorrect evaluation of pelvic vein diameter due to vasospasm $[1,19]$. Fortunately, neither complications were observed in our study, which likely might be attributed to the physicochemical property of Glubran-2 and the knowledge and experience of clinicians in employing Glubran-2. Moreover, ovarian vein embolization was found no significant changes in follicle stimulating hormone, luteinizing hormone, or estradiol levels, which were thought to be unassociated with pregnancy $[1,18,20]$. Data revealed that Glubran-2 used in postpartum hemorrhage was without adversely affect in uterine function when embolizing uterine arteries [21]. However, there is no enough evidences whether reproductive function is affected in our study, interesting, two pa-tients who underwent Glubran-2 embolization of the ovarian vein gave birth to a healthy baby.

In present study, all patients experienced coaxial catheter technique. Before micro- coils used, a compatible 2.4-F progreat microcatheter was coaxially positioned, insuring the microcatheter tip across dilated ovarian vein as close as possible into the target varicose veins of the utero-ovarian plexus, then Glubran-2 was injected under withdraw from distal insufficient tributary branches to proximal trunk to achieve the precise occlusion of the origin of the leak. Microcatheter employed in present study had a low adverse event of instant adhesion, which is likely attributed to the hydrophilic surface coating of microcatheter tip. Even facing the condition of adhesion of microcatheter, it can be drawn back safely under the support 
protection of Cobra catheter to avoid colloidal overflow. It is noteworthy that, in experience, Glubran-2 appears to be more economical compared to microcoils used alone, as well as has an advantage of no metal residual in patients' body, in the future, they can undergo CT and MRI investigations avoiding image quality affecting.

The present study exists a number of limitations. First, pain levels are commonly subjective, since CPP in our study were evaluated mainly according to the VAS, which might lead to a basis; Second, our study was relatively small and retrospective, with all its inherent limitations, a multi-institutional prospective study may be required to determine the conclusions. Third, the aim of present study mainly focuses on evaluate preliminary outcome of ovarian vein embolization with Glubran-2 for treatment of CPP secondary to PCS, no comparison is conducted among Glubran-2 and conventional embolic materials, which may be needed to show subsequently. Despite above all, to our best knowledge, the present study may be by far a solely study and the largest case series regarding embolization using Glubran-2 as the embolic material for evaluating CPP secondary to PCS.

In conclusion, ovarian vein embolization with NBCA Glubran-2 is a feasible and safe treatment of CPP secondary to PCS. In particularly, it appears to be a potential and attractive alternative when patients with the desires of meaningful symptom relief and reproduction. Glubran-2 is effective in achieving complete embolization of pelvic varicosities through an ovarian vein, without the risk of the migration of embolic material. Further large studies are warranted to confirm the findings.

\section{Declarations}

Ethics approval and consent to participate The study protocol was reviewed and approved by the institutional review board (IRB) of the Nanjing First Hospital, Nanjing Medical University (Nanjing, China). In addition, the study was performed in accordance with the Declaration of Helsinki and the Ethical Guidelines for Clinical Studies.

Consent for publication Not applicable.

Availability of data and materials The datasets generated and analyzed during the current study are not publicly available as the experimental data are related to other experiments that are progressing, but are available from the corresponding author on reasonable request.

Competing interests The authors of this manuscript declare no relationships with any companies whose products or services may be related to the subject matter of the article. The content of the manuscript is original, and it has not been published or accepted for publication.

Funding This work was supported by the National Natural Science Foundation of China (8187 1463).

Authors' contributions MFG contributed to data collection, manuscript writing/ editing. XH and BXZ contributed to project development, data collection, data analysis. JK contributed to project development, data collection, data analysis, manuscript editing. HBS contributed to project development.

Acknowledgements I will thank my wife Qing Zhao, during she was busy fighting against evil COVID-19, i was given a lonely condition and enough time to finish this manuscript, as well as moral encouragements from her. May health along with everyone, and i expect that this manuscript can provide you with little help.

\section{References}

1. Antignani PL, Lazarashvili Z, Monedero JL, et al. Diagnosis and treatment of pelvic congestion syndrome: UIP consensus document. Int Angiol. 2019;38:265-83.

2. Borghi C, Dell'Atti L. Pelvic congestion syndrome: the current state of the literature. Arch Gynecol Obstet. 2016;293:291-301. 
3. Lazarashvili Z, Antignani PL, Monedero JL. Pelvic congestion syndrome: prevalen-ce and quality of life. Phlebolymphology. 2016;23:123-6.

4. Champaneria $\mathrm{R}$, Shah $\mathrm{L}$, Moss $\mathrm{J}$, et al. The relationship between pelvic vein incompetence and chronic pelvic pain in women: systematic reviews of diagnosis and treatment effectiveness. Health Technol Assess. 2016;20:1-108.

5. Gloviczki P, Comerota AJ, Dalsing MC, et al. The care of patients with varicose veins and associated chronic venous diseases: clinical practice guidelines of the society for vascular surgery and the American venous forum. J Vasc Surg. 2011;53:2S-48S.

6. Black CM, Thorpe K, Venbrux A, et al. Research reporting standards for endo-vascular treatment of pelvic venous insufficiency. J Vasc Interv Radiol. 2010;21:796-803.

7. Daniels JP, Champaneria R, Shah L, et al. Effectiveness of embolization or sclero-therapy of pelvic veins for reducing chronic pelvic pain: a systematic review. J Vasc Interv Radiol. 2016;27:1478-86.

8. Kwon SH, Oh JH, Ko KR, Park HC, Huh JY. Transcatheter ovarian vein embolize-tion using coils for the treatment of pelvic congestion syndrome. Cardiovasc Inter-vent Radiol. 2007;30:655-61.

9. Leatherby RJ, Harries P, Shah SS. The management of pelvic congestion syndrome a word of caution. J Obstet Gynaecol. 2020;40:283-4.

10. Yonemitsu T, Kawai N, Sato M, et al. Evaluation of transcatheter arterial emboliza-tion with gelatin sponge particles, microcoils, and N-butyl Cyanoacrylate for acute arterial bleeding in a coagulopathic condition. J Vasc Interv Radiol. 2009;20:1176-87.

11. Angle JF, Siddiqi NH, Wallace MJ, et al. Quality improvement guidelines for percu-taneous transcatheter embolization: Society of Interventional Radiology Standards of Practice Committee. J Vasc Interv Radiol. 2010;21:1479-86.

12. Laborda A, Medrano J, de Blas I, et al. Endovascular treatment of pelvic congestion syndrome: Visual Analog Scale (VAS) long-term follow-up clinical evaluation in 202 Patients. Cardiovasc Intervent Radiol. 2013;36:1006-14.

13. Brown CL, Rizer M, Alexander R, Sharpe EE 3rd, Rochon PJ. Pelvic congestion syndrome: systematic review of treatment success. Semin Intervent Radiol. 2018;35:35-40.

14. Meissner $\mathrm{MH}$, Gibson K. Clinical outcome after treatment of pelvic congestion syndrome: sense and nonsense. Phlebology. 2015;30:73-80.

15. Gavrilov SG, Turischeva O0. Conservative treatment of pelvic congestion syndro-me: indications and opportunities. Curr Med Res Opin. 2017;33:1099-103.

16. Hansrani V, Abbas A, Bhandari S, et al. Transvenous occlusion of incompetent pelvic veins for chronic pelvic pain in women: a systematic review. Eur J Obstet Gynecol Reprod Biol. 2015;185:156-63.

17. Scultetus AH, Villavicencio JL, Gillespie DL, Kao TC, Rich NM. The pelvic venous syndromes: analysis of our experience with 57 patients. J Vasc Surg. 2002;36:881-8.

18. Kim HS, Malhotra AD, Rowe PC, Lee JM. V enbrux AC. Embolotherapy for pelvic congestion syndrome: long-term results. J Vasc Interv Radiol. 2006;17:289-97.

19. van der Vleuten CJ, van Kempen JA, Schultze-Kool LJ. Embolization to treat pelvic congestion syndrome and vulval varicose veins. Int J Gynaecol Obstet. 2012;118:227-30.

20. Liu J, Han L, Han X. The effect of a subsequent pregnancy after ovarian vein embolization in patients with infertility caused by pelvic congestion syndrome. Acad Radiol. 2019;26:1373-7.

21. Cantasdemir M, Adaletli I, Cebi D, et al. Emergency endovascular embolization of traumatic intrarenal arterial pseudoaneurysms with N-Butyl Cyanoacrylate. Clin Radiol. 2003;58:560-5.

\section{Figures}




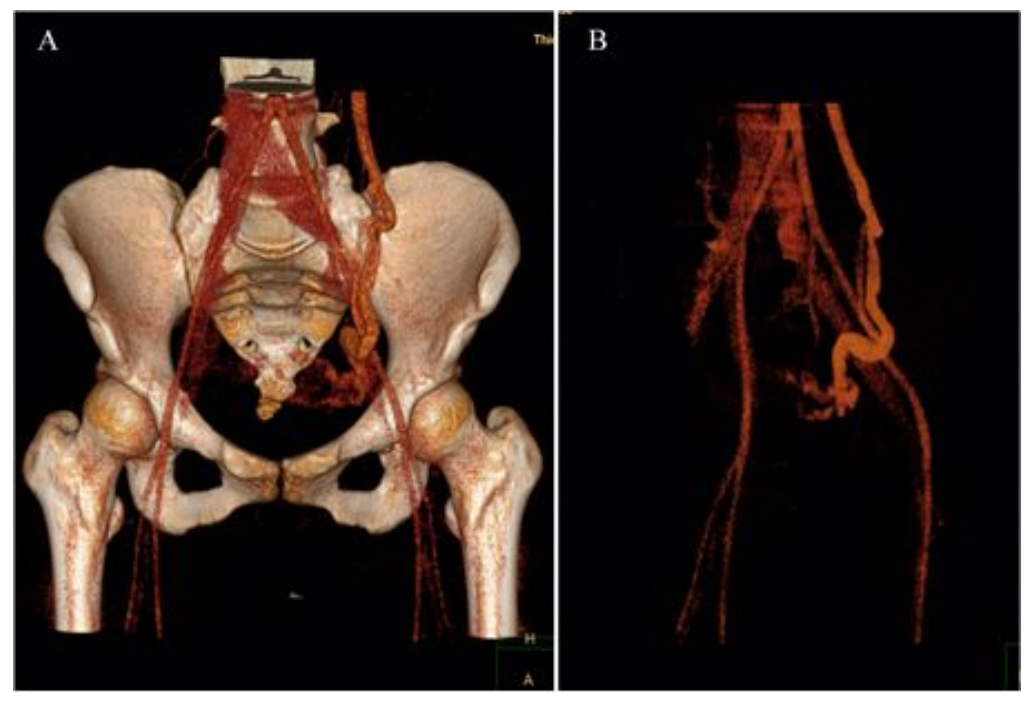

\section{Figure 1}

Three dimensions reconstruction of enhanced CT revealed varicose gonadal veins. (A) Anteroposterior. (B) Loxosis.
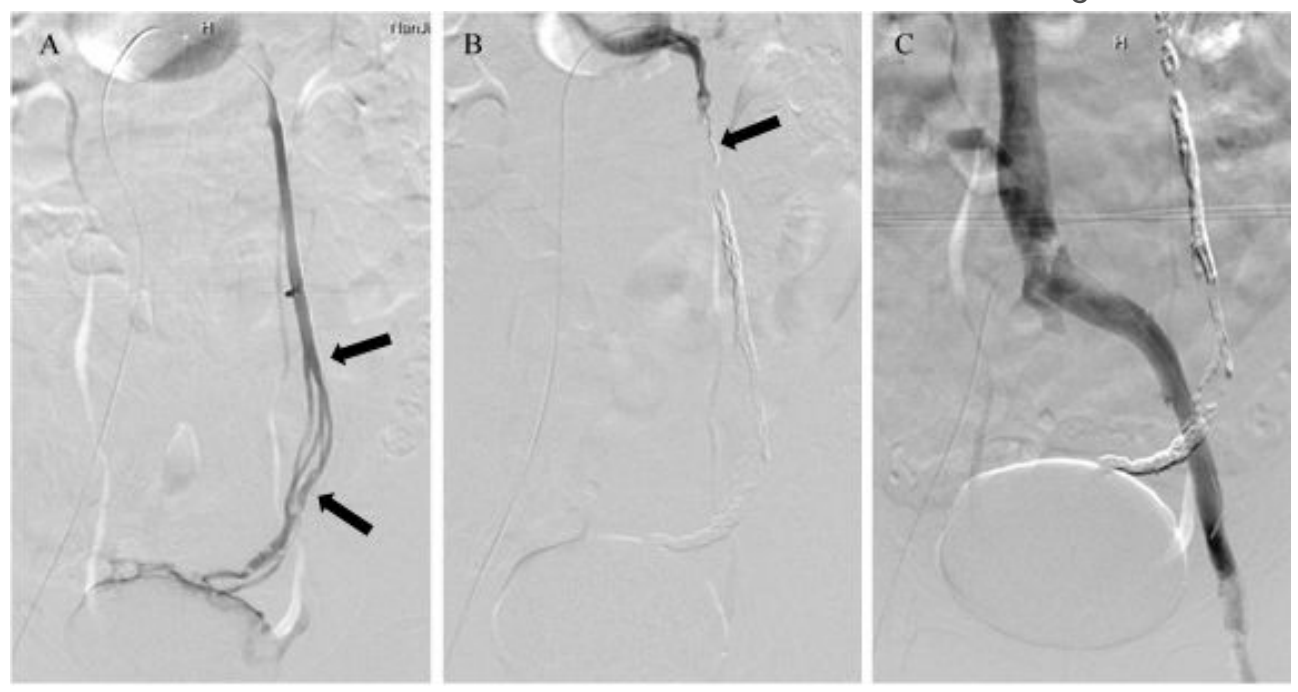

\section{Figure 2}

A 59-year-old women with chronic pelvic pain secondary to pelvic congestion syn- drome. (A) Selective left ovarian vein venography demonstrating retrograde flow in a dilated incompetent gonadal vein (black arrows). (B) Ovarian vein embolization with Glubran-2, the varicose gonadal veins were complete occluded, and the opening of ovarian vein trunk was sealed with microcoils (black arrow). (C) Final venography of left iliac vein after embolization revealed no reflux flow draining into utero-ovarian plexus. 

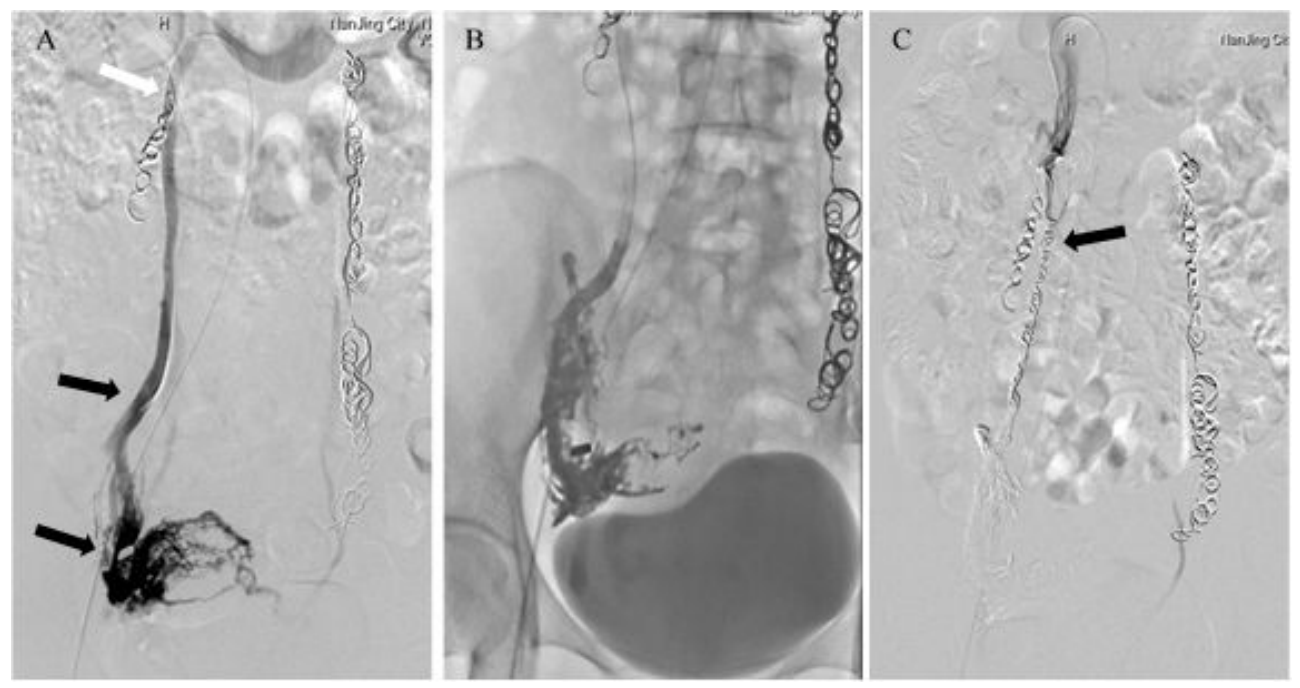

\section{Figure 3}

A 38-year-old women encountered the second ovarian vein embolization using Glu- bran-2 due to chronic pelvic pain recurrence one month after initial embolization with microcoils. (A) Right ovarian vein venography demonstrating reflux flow in a dilated varicose ovarian vein and uterine veins engorgement extending across the midline (black arrows). Ovarian vein is consisted of multiply trunks and the tail of microcoils initially used were partially inserted into the main trunk (white arrow). (B) Ovarian vein embolization with Glubran-2, the varicose gonadal veins were complete occluded. (C) The opening of ovarian vein trunk was sealed with microcoils (black arrow), no retrograde flow was seen in the right ovarian vein postembolization with Glubran-2 and sealing of microcoils.

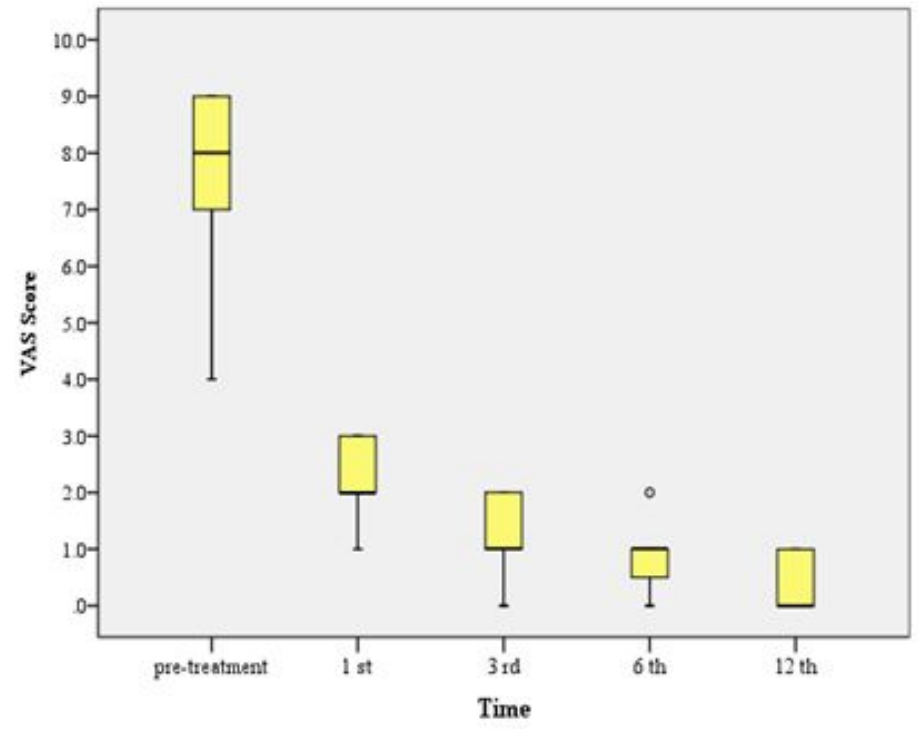

\section{Figure 4}

Progression of CPP evaluated by VAS score during the 12-month follow-up Note: $\mathrm{CPP}=$ chronic pelvic pain, VAS $=$ visual analog scale 